\title{
Comparative Study of Matrix-Assisted Laser Desorption/Ionization Mass Spectrometry and Culture Test for Candida Identification
}

\author{
Yoriko Kubota $^{1 *}$, Chieko Taguchi², Masanori Saito ${ }^{3}$, Noriko Shinozaki-Kuwahara ${ }^{3}$, \\ Tomoko Suzuki ${ }^{1}$, Masaaki Suemitsu ${ }^{1}$, Mitsuko Nakayama ${ }^{1}$, Tadahiko Utsunomiya ${ }^{1}$, \\ Hiroyasu Endo4, Kayo Kuyama1 \\ ${ }^{1}$ Department of Pathology, Nihon University School of Dentistry at Matsudo, Chiba, Japan \\ ${ }^{2}$ Department of Microbiology and Immunology, Nihon University School of Dentistry at Matsudo, Chiba, Japan \\ ${ }^{3}$ Department of Community Oral Health, Nihon University School of Dentistry at Matsudo, Chiba, Japan \\ ${ }^{4}$ Department of Oral Diagnosis, Nihon University School of Dentistry at Matsudo, Chiba, Japan \\ Email: ^kubota_river@yahoo.co.jp
}

How to cite this paper: Kubota, Y., Taguchi, C., Saito, M., Shinozaki-Kuwahara, N., Suzuki, T., Suemitsu, M., Nakayama, M., Utsunomiya, T., Endo, H. and Kuyama, K. (2019) Comparative Study of Matrix-Assisted Laser Desorption/Ionization Mass Spectrometry and Culture Test for Candida Identification. Open Journal of Stomatology, 9, 295-306.

https://doi.org/10.4236/ojst.2019.912030

Received: October 20, 2019

Accepted: December 14, 2019

Published: December 17, 2019

Copyright () 2019 by author(s) and Scientific Research Publishing Inc. This work is licensed under the Creative Commons Attribution International License (CC BY 4.0).

http://creativecommons.org/licenses/by/4.0/ (c) (i) Open Access

\begin{abstract}
Background: A new microorganism identification method using matrix-assisted laser desorption/ionization mass spectrometry (MALDI-TOF MS) has been developed, however, reports on its use for delineating Candida spp. are scarce. Objectives: The purpose of this study was to compare the identification accuracy of mixed infection between culture test and MALDI-TOF MS. Materials and Methods: Eighty-nine denture wearers (average 74.0 \pm 9 years) were selected. Specimens were immediately inoculated onto selective medium for CHROMagar $^{\text {rm }}$ Candida, and were also carried out using MALDI-TOF MS. The distribution frequencies of them were analyzed. Results: The numbers and rates of detection/non-detection by MALDI-TOF MS of genus Candida were $58 / 31(65.2 \% / 34.8 \%)$, respectively. Infection types were single infection in 34 (38.2\%), mixed infection in $24(27.0 \%)$, and non-infection in $31(34.8 \%)$ cases. Concerning the single infection, $C$. albicans was the most predominant (58.8\%), followed by C. parapsilosis (17.6\%), C. glabrata (14.7\%), C. tropicalis (5.9\%), and C. krusei (2.9\%). As for the mixed infection, the most frequent combination was $C$. albicans and $C$. glabrata (50.0\%), followed by $C$. albicans and C. parapsilosis (29.2\%), C. albicans and C. tropicalis (8.3\%), C. glabrata and C. tropicalis (4.2\%), C. albicans, C. glabrata, and $C$. parapsilosis (4.2\%), and $C$. albicans, C. parapsilosis, and C. glabrata (4.2\%). There were four MALDI-TOF MS positive results that were negative by the culture test. Conversely, there were six MALDI-TOF MS negative results that were positive by the culture test. The sensitivity and specificity of MALDI-TOF MS were 0.929 and 0.840 ,
\end{abstract}


respectively. The concordance rate of genus Candida was 0.644 , indicating substantial agreement. Conclusion: Candida infection is complicated by disease type and oral cavity environment changes due to aging. A rapid microorganism detection method, such as MALDI-TOF MS, will be helpful to quickly determine the causative pathogen in dental infections.

\section{Keywords}

Candida, Culture Test, MALDI-TOF MS, Non-C. Albicans Candida (NCAC)

\section{Introduction}

Candida is a large genus of ascomycetous yeast, consisting of about 150 species, and more than 20 species have clinical importance [1]. Candida spp. are found in the oral cavity of $25 \%-50 \%$ of healthy individuals, including adults and children. When only denture wearers are considered, these frequencies increase to $60 \%-100 \%$ [2]. Moreover, wearing a dental prosthesis such as a removable denture [3] or removable orthodontic appliance [4] enhances oral candidal colonization and predisposes the wearer to oral candidiasis.

Candida albicans is the most frequently isolated species and the causative species of almost 70\% of Candida infections [5] [6]. Although C. albicans is a well-known colonizer and pathogen of the oral mucosa, non- $C$. albicans Candi$d a(\mathrm{NCAC})$ is increasingly encountered [7] [8] [9] and its emerging role in human infections has gained attention [10] [11]. This rising number in infections caused by NCAC species may reflect both the improvement in diagnostic methodologies and the superior capability of NCAC species to persist in the host compared with $C$. albicans. Moreover, in human mixed candidiasis, NCAC species demonstrate high antifungal resistance profiles 10).

CHROMagar $^{\text {Tax }}$ Candida is a very useful medium to distinguish Candida spp. such as $C$. albicans, C. tropicalis, C. krusei, and C. glabrata, which account for almost $90 \%$ of all clinical yeastisolates, including dental samples [12] [13]. However, CHROMagar ${ }^{\mathrm{ma}}$ Candida cannot definitely distinguish C. albicans from C. dubliniensis [14] [15], which is more often isolated from HIV-positive patients. In addition, the accuracy of CHROMagar ${ }^{\text {Ta }}$ to distinguish particular Candida spp. from mixed infection is poorly examined. Namely, insufficient evidence is available about the quality of CHROMagar ${ }^{\text {ra }}$ Candida testing in patients with various underlying conditions. A new microorganism identification method using matrix-assisted laser desorption/ionization mass spectrometry (MALDITOF MS) has been developed, however, reports on its use for delineating Candida spp. are scarce [16] [17] [18].

Therefore, the purposes of this study were to compare the identification accuracy of mixed infection between CHROMagar ${ }^{\text {rm }}$ Candida and MALDI-TOF MS in older denture wearers, and to highlight gaps in knowledge and the justification for the present work about candidal distribution. 


\section{Material and Methods}

\subsection{Subjects}

Eighty-nine denture wearers (49 males, 40 females) who had visited Kubota Dental Hospital for denture adjustment/checkup from August 2017 to September 2018 were selected for inclusion in this study. The mean age and standard deviation of subjects were $74.0 \pm 9$ years (range, 43 - 99 years). Inclusion criteria were individuals who: 1) were wearing full/partial dentures; 2 ) had data for oral examination and microbiological tests; 3 ) had agreed to the study. Exclusion criteria were individuals who: a) were taking antifungal agents or using antiseptic mouthwashes; b) were taking any medication known to predispose them to oral candidiasis; c) had a medical history that revealed any disease or medical condition (such as diabetes mellitus or anemia) that predisposed them to oral candidiasis or promoted candidal carriage in the 6 weeks before the study; or d) for whom necessary data were lacking. None of the included subjects had signs or symptoms suggestive of candidal paronychia or dermatologic fungal infection.

\subsection{Microbiological Examination}

1) Sample collection

To reduce the bias in sampling procedures, microbiological samples were collected from all patients by swabbing denture mucosal surfaces 10 times with a cotton swab dipped in sterile purified water approximately 2 hours or more after breakfast eating.

2) Culture test using CHROMagar ${ }^{\mathrm{Tm}}$

Specimens were then immediately inoculated onto selective medium for Candida, CHROMagar ${ }^{\mathrm{Tx}}$ Candida (KANTO KAGAKU, Tokyo, Japan). After aerobic incubation for $24-48$ hours at $25^{\circ} \mathrm{C}$, Candida spp. were manually measured and enumerated by colony morphology and color. According to the manufacturer's instructions, $C$. albicans colonies are distinguished by a distinctive greencolor, $C$. glabrata colonies exhibit a purple to pale pink color, C. tropicalis colonies have a dark blue color with pink edge, $C$. krusei colonies are rough with pale pink centers and white edges, and $C$. parapsilosis colonies present a white or pale pink color. After the 48-hour incubation on CHROMagar ${ }^{\mathrm{mix}}$, incubation was continued for more than 2 weeks at $37^{\circ} \mathrm{C}$ to confirm not to culture.

3) MALDI-TOF MS analysis

Species determination of all samples was also carried out using MALDI-TOF MS (BD Bruker MALDI Biotyper ${ }^{\mathrm{mw}}$; BD). Fungal colonies were thinly applied to the target plate using a toothpick, $1 \mu \mathrm{l}$ of matrix was added, and samples were measured according to the manufacturer's protocol. Application of a sample is one time, and MALDI Biotyper measures the strain several times and calculates average value of each peak of a mass spectrum and the strength. Acquired spectrum were expressed as a score value by phylogenetic tree analysis using a pattern matching spectral library. The case where the score value was 2.0 or more was taken as the identification result at the bacterial species level. 


\subsection{Statistical Analysis}

The distribution frequencies of Candida spp. of culture test and MALDI-TOF MS were analyzed. The coincidence rate of culture testing and MALDI-TOF MS was examined using the kappa coefficient with 95 confidence interval [CI]. The software used by statistical analysis of this research was Bell Curve for Excel.

\subsection{Compliance with Ethical Standards}

Informed consent was obtained from all individuals included in the study. All procedures in studies involving human participants were conducted in accordance with the ethical standards of the Committee on Studies Involving Human Beings of Nihon University School of Dentistry at Matsudo (EC-15-14-033-1) and with the 1964 Helsinki declaration and its later amendments or comparable ethical standards.

\section{Results}

\subsection{Subject Characteristics}

Characteristics of subjects in the present study were shown in Table 1. Subjects consisted of 49 males and 40 females (average age $74.1 \pm 9.1$ ). Denture types included upper full denture $(n=25)$, lower full denture $(n=2)$, upper partial denture $(\mathrm{n}=47)$, and lower partial denture $(\mathrm{n}=15)$. The mean number of artificial teeth indentures was 9.88. Gross findings of mucosal surfaces of the denture base identified 18 cases (20.2\%) of denture stomatitis (DRS) and 71 cases (79.8\%) with no evidence of DRS. Concerning DRS, 13 cases $(72.2 \%)$ had reddish mucosa, 3 cases had (16.7\%) whitish mucosa, and 2 cases (11.1\%) had mixed red and white mucosa.

Table 1. Characteristics of subjects.

\begin{tabular}{ccc}
\hline Item & Character & \\
\hline \multirow{2}{*}{ Sex } & Male & 49 \\
& Female & 40 \\
& Total & 89 \\
Ave. $\pm \mathrm{SD}^{*}$ & Male & $74.1 \pm 9.0$ \\
& Female & $74.1 \pm 9.0$ \\
& Total & $74.1 \pm 9.1$ \\
Denture types & Upper full denture & 25 \\
& Lower full denture & 2 \\
Artificial teeth & Upper partial denture & 47 \\
& Lower partial denture & 15 \\
& Average number & 9.88 \\
Denture stomatitis & Reddish mucosa & 13 \\
& Whitish mucosa & 3 \\
& Mixed red and white & 2 \\
& Total & 18 \\
\hline
\end{tabular}

*: Average age \pm standard deviation. 


\subsection{Microbiological Distribution}

The results of microbiological distribution were listed on the cross tabulation in Table 2.

1) Culture test using CHROMagar ${ }^{\mathrm{m}}$

Candida colonies were detected from the denture mucous membrane of 59 (66.3\%) subjects. Infection types included single infection in $34(57.6 \%)$ subjects and mixed infection in 25 (42.4\%) subjects. Concerning single infection, Candi$d a$ distribution by colony morphology and color were as follows: $C$. albicans in 24 (70.6\%), C. parapsilosis in 4 (11.8\%), C. glabrata in 4 (11.8\%), and C. tropicalis in $2(5.9 \%)$ cases. As for the 25 cases of mixed infection, $C$. albicans and $C$. glabrata were identified in 15 (60.0\%) cases, C. albicans and C. parapsilosis were identified in $5(20.0 \%)$ cases and C. albicans and C. tropicalis were identified in 3 (12.0\%) case, C. albicans and C. krusei in 1 (4.0\%) and C. albicans, C. parapsilosis, and C. tropicalis were identified in $1(4.0 \%)$ case.

2) MALDI-TOF MS analysis

The numbers and rates of detection/non-detection by MALDI-TOF MS of genus Candida were 58/31 (65.2\%/34.8\%), respectively. Infection types were single infection in 34 (38.2\%), mixed infection in 24 (27.0\%), and non-infection in 31 (34.8\%) cases. Concerning the 34 cases of single infection, $C$. albicans was the

Table 2. Cross tabulation between culture test (CHROMagar $\left.{ }^{\mathrm{TM}}\right)$ and MALDI-TOF MS analysis.

\begin{tabular}{|c|c|c|c|c|c|c|c|c|c|c|c|c|c|c|c|c|c|}
\hline \multicolumn{18}{|c|}{ MALDI-TOF MS } \\
\hline & & C. $a^{*}$ & $C . g^{* *}$ & $C . k^{* * *}$ & $C \cdot p^{\dagger}$ & C. $t^{\dagger \dagger}$ & $\begin{array}{l}\text { C.a, } \\
\text { C.g }\end{array}$ & $\begin{array}{l}\text { C.a, } \\
\text { C.k }\end{array}$ & $\begin{array}{l}\text { C.a, } \\
\text { C.p }\end{array}$ & $\begin{array}{l}\text { C.a, } \\
\text { C.t }\end{array}$ & $\begin{array}{l}\text { C.g, } \\
\text { C.t }\end{array}$ & $\begin{array}{l}\text { C.a, } \\
\text { C.g, }\end{array}$ & $\begin{array}{l}\text { C.a, } \\
\text { C.p, }\end{array}$ & Non-detection & Total & & \\
\hline \multirow{15}{*}{ 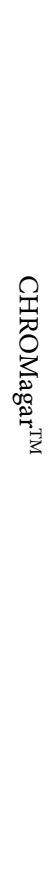 } & C.a & 17 & 1 & 0 & 0 & 1 & 0 & 0 & 0 & 0 & 0 & 0 & 0 & 5 & 24 & 70.8 & \\
\hline & C.g & 0 & 4 & 0 & 0 & 0 & 0 & 0 & 0 & 0 & 0 & 0 & 0 & 0 & 4 & 100.0 & \\
\hline & C.k & 0 & 0 & 0 & 0 & 0 & 0 & 0 & 0 & 0 & 0 & 0 & 0 & 0 & 0 & - & \\
\hline & C.p & 0 & 0 & 1 & 3 & 0 & 0 & 0 & 0 & 0 & 0 & 0 & 0 & 0 & 4 & 75.0 & \\
\hline & C.t & 0 & 0 & 0 & 0 & 1 & 0 & 0 & 1 & 0 & 0 & 0 & 0 & 0 & 2 & 50.0 & \\
\hline & C.a, C.g & 0 & 0 & 0 & 0 & 0 & 11 & 0 & 2 & 0 & 1 & 1 & 0 & 0 & 15 & 73.3 & \\
\hline & C.a, C.k & 0 & 0 & 0 & 0 & 0 & 0 & 0 & 1 & 0 & 0 & 0 & 0 & 0 & 1 & 0.0 & \\
\hline & C.a, C.p & 0 & 0 & 0 & 1 & 0 & 0 & 0 & 2 & 0 & 0 & 1 & 0 & 1 & 5 & 40.0 & \\
\hline & C.a, C.t & 0 & 0 & 0 & 1 & 0 & 0 & 0 & 0 & 1 & 1 & 0 & 0 & 0 & 3 & 33.3 & \\
\hline & C.g, C.t & 0 & 0 & 0 & 0 & 0 & 0 & 0 & 0 & 0 & 0 & 0 & 0 & 0 & 0 & - & \\
\hline & C.a, C.g, C.p & 0 & 0 & 0 & 0 & 0 & 0 & 0 & 0 & 0 & 0 & 0 & 0 & 0 & 0 & - & \\
\hline & C.a, C.p, C.t & 0 & 0 & 0 & 0 & 0 & 0 & 0 & 1 & 0 & 0 & 0 & 0 & 0 & 1 & 0.0 & \\
\hline & Non colonies & 3 & 0 & 0 & 1 & 0 & 1 & 0 & 0 & 0 & 0 & 0 & 0 & 25 & 30 & 83.3 & \\
\hline & Total (n) & 20 & 5 & 1 & 6 & 2 & 12 & 0 & 7 & 1 & 2 & 2 & 0 & 31 & 89 & & \\
\hline & $\begin{array}{l}\text { Concordance } \\
\text { rate }(\%)\end{array}$ & 85.0 & 80.0 & 0.0 & 50.0 & 50.0 & 91.7 & - & 28.6 & 100.0 & 0.0 & 0.0 & - & 80.6 & & 64 & 71.9 \\
\hline
\end{tabular}

C.a*: Candida albicans, C. $g^{* *}:$ Candida glabrata; C.k ${ }^{* *}:$ Candida krusei; $C . p^{\dagger}:$ Candida parapsilosis; C.t $t^{\dagger}$ Candida tropicalis. 
most predominant $(\mathrm{n}=20,58.8 \%)$, followed by $C$. parapsilosis $(\mathrm{n}=6,17.6 \%), C$. glabrata $(\mathrm{n}=5,14.7 \%)$, C. tropicalis $(\mathrm{n}=2,5.9 \%)$, and C. krusei $(\mathrm{n}=1,2.9 \%)$. As for the 24 cases of mixed infection, the most frequent combination was $C$. albicans and $C$. glabrata $(\mathrm{n}=12,50.0 \%)$, followed by $C$. albicans and $C$. parapsilosis $(\mathrm{n}=7,29.2 \%), C$. glabrata and $C$. tropicalis $(\mathrm{n}=2,8.3 \%), C$. albicans, $C$. glabrata, and $C$. parapsilosis $(\mathrm{n}=2,8.3 \%)$, and $C$. albicans and $C$. tropicalis $(\mathrm{n}=1$, $4.2 \%)$.

3) Comparison of culture test and MALDI-TOF MS results

There were four MALDI-TOF MS positive results ( $C$. albicans, $\mathrm{n}=2$; $C$. albicans and $C$. glabrata, $\mathrm{n}=1$; and $C$. albicans and Penicillium spp., $\mathrm{n}=1$ ) that were negative by the culture test. Conversely, there were six MALDI-TOF MS negative results (sample degeneration) by drying, $\mathrm{n}=4$; Aspergillus spp., $\mathrm{n}=1$; and $C$. albicans and Penicillium spp., $\mathrm{n}=1$ ) that were positive by the culture test.

\subsection{Statistical Results}

The sensitivity and specificity of MALDI-TOF MS were 0.929 and 0.840 , respectively. The concordance rate (kappa coefficient) of genus Candida was 0.644, indicating substantial agreement [19]. Table 1 shows all concordance rates for single and mixed infections of all Candida spp. and combinations of mixed infection. Concordance rates of single infection were as follows: $C$. albicans $85.0 \%, C$. glabrata $80.0 \%$, C. parapsilosis $50.0 \%$, C. tropicalis $50.0 \%$, and C. krusei $0.0 \%$. Furthermore, concordance rates of mixed infection were as follows: $C$. albicans and $C$. tropicalis $100.0 \%, C$. albicans and $C$. glabrata $91.7 \%, C$. albicans and $C$. parapsilosis $28.6 \%, C$. glabrata and $C$. tropicalis $0.0 \%$, and C. albicans, C. glabrata, and C. parapsilosis $0.0 \%$. In addition, the concordance rate of Candida negative was $80.6 \%$.

\section{Discussion}

The elderly are known to be more vulnerable to fungal infections because of underlying conditions, such as chronic diseases, medications, poor oral hygiene, reduced salivary flow, and immune system impairment [20] [21] [22]. Furthermore, fungi are isolated not only from the oral cavity but also from tissue-fitting surfaces and outer surfaces of dentures [21]. Therefore, in this study, we evaluated two Candida spp. detection methods, culture test and MALDI-TOF MS, in older denture wearers (mean age, 74 years) without subjective symptoms.

C. albicans is the most frequently isolated Candida spp. as a colonizer and pathogen of the oral mucosa but reports on the appearance of NCAC are increasing [23] [24] [25]. This shift towards NCAC species is mainly due to severe immunosuppression, use of broad-spectrum antibiotics, and empirical use of antifungal drugs [7] [8] [9]. Nevertheless, NCAC infections are usually indistinguishable based on symptoms alone due to similarities in clinical presentations. Consequently, the need for improvement of NCAC mixed infection diagnostic methodologies has increased. 
The present study detected $66.3 \%$ and $65.2 \%$ of NCAC by culture test and MALDI-TOF MS, respectively, and these values were higher than that of a previous study (58.5\%) [26]. CHROMagar ${ }^{\mathrm{Tm}}$ is a commercial product that was developed for the isolation and presumptive identification of specimens containing mixtures of yeast species [27]. CHROMagar ${ }^{\mathrm{ma}}$ Candida is a very useful medium to distinguish Candida spp. with different morphologies and colors, which account for almost $90 \%$ of all clinical yeastisolates, including dental samples [12] [13]. The specificity and sensitivity of C. albicans, C. tropicalis, and C. krusei identification exceed 99\% [27]. However, changes in the oral environment, such as during severe immunosuppression, increase the number of NCAC species, and the sensitivity of CHROMagar ${ }^{\mathrm{Tm}}$ Candida in detecting mixed infections with NCAC has not been sufficiently examined.

Bacterial identification by MALDI-TOF MS in outsourced inspection has gained attention as a rapid diagnostic method for clinical use. Advantages of MALDI-TOF MS included small bacterial sample needed, easy preprocessing, and high spectral analyses. Numerous species have been identified at the genus level by MALDI-TOF MS; particularly, the same number of species have been identified for genera Candida and Cryptococcus [28] [29]. However, MALDITOF MS data on Candida genus remain scarce.

In the present study, MALDI-TOF MS analyses demonstrated that $C$. albicans (58.8\%) remained the predominant species in oral candidiasis patients respectively, followed by C. parapsilosis (17.6\%), C. glabrata (14.7\%), C. tropicalis (5.9\%), and C. krusei (2.9\%). Although the species distribution was similar to those of other reports [5] [6], the proportion of NCAC was higher [26] [30] [31]. In the previous two decades, $C$. albicans represented over $80 \%$ of all human candidiasisisolates [32]. In this study, C. glabrata and C. parapsilosis proportions were particularly high. C. glabrata and C. parapsilosis have emerged as significant nosocomial pathogens. C. glabrata is associated with immunosuppressive and antimicrobial treatments [10] [33], while C. parapsilosis is associated with invasive procedures [11]. The higher proportions of NCAC species observed in the present study may be explained by the study cohort characteristics (older subjects with chronic diseases) and increased immunosuppression by advanced medical treatment. Thus, the oral cavities of hospitalized older adults contain greater species diversity than previously thought and is not limited to typical Candida species.

Although most Candida-positive carriers (62.9\%) possessed only one Candida spp., $37.1 \%$ of subjects harbored more, which was similar to the report by Lockhart et al. [22]. Although Candida spp. are generally not very aggressive, they may cause infection if the immune system is impaired or if an environmental niche becomes available. Mixed infection involving NCAC has been reported in recent years. Specifically, $36.6 \%$ of cases were $C$. parapsilosis species mixed. Furthermore, C. tropicalis, C. glabrata species mixed, and C. krusei comprised $35.4 \%, 24.3 \%$, and $3.7 \%$ of all isolates, respectively [34]. In the present study, the 
most frequent combination was $C$. albicans and C. glabrata (50.0\%), and 87.5\% of mixed infection was a combination of C. albicans and NCAC. Accordingly, we focused the results of our study on the distribution of Candida species. The microbiological examination showed that the most frequently isolated species were $C$. albicans, $C$. glabrata, and C. parapsilosis. The latter species belong to NCAC species that have recently caused systemic candidiasis, and fatal cases of C. glabrata infection have been reported [35] [36].

The kappa coefficient between culture test and MALDI-TOF MS was 0.644, which indicated substantial agreement 19). The highest concordance rate of single infection was $C$. albicans (85.0\%), followed by C. glabrata (80.0\%). These high concordance rates may be explained by the ease of distinguishing $C$. albicans and C. glabrata colonies by color (green and deep purple, respectively). Conversely, C. parapsilosis, C. tropicalis, and C. krusei, which have similar colony colors, presented low concordance rates. In addition, the concordance rate of Candida negative was $80.6 \%$. Furthermore, concerning mixed infections, $C$. albicans and C. parapsilosis, C. glabrata and C. tropicalis, and C. albicans, C. glabrata, and C. parapsilosis showed low or non-concordance rates. We speculate that the number of fungi on agar plates was large, colonies were fused due to mixed infection, and identification by colony color is subjective. However, these issues do not affect identification by MALDI-TOF MS. Furthermore, our findings suggested that degeneration due to fungal death leads to overdiagnosis by the culture test. The sensitivity and specificity rates of MALDI-TOF MS were high, even in cases of mixed infection. It was speculated that the reason for the very few misidentifications was caused by spectral pattern matching for each Candida species. More than one strain by the same species was registered in the spectral library, so even if diversity with the spectral pattern existed between strains, it should be possible to correctly identification. With the increase in NCAC and multiple infections, the value of MALDI-TOF MS application is recognized for Candida identification. In addition, there is a need to improve the criteria for simple CHROMagar ${ }^{\mathrm{m}}$ culture testing for diagnosis of oral infections.

In conclusion, Candida mixed infection was observed in $27.0 \%$ of older Japanese denture wearers. Candida infection is complicated by disease type and oral cavity environment changes due to aging. A rapid microorganism detection method, such as MALDI-TOF MS, will be helpful to quickly determine the causative pathogen in dental infections.

\section{Conclusions}

The following could be concluded in the present study:

1) The numbers and rates of detection by MALDI-TOF MS of genus Candida were $65.2 \%$ of elder denture wearers.

2) The rates of single and mixed infection were $38.2 / 27.0 \%$, respectively.

3) C. albicans (58.8\%) of single and C. albicans and C. glabrata (50.0\%) of mixed infection were the most predominant. 
4) The sensitivity and specificity of MALDI-TOF MS were 0.929 and 0.840 , respectively.

\section{Acknowledgements}

This research was supported by scientific support from Dr. YuzoTsuyuki and JSPS KAKENHI Grant Number JP 18K07000.

\section{Conflicts of Interest}

The authors declare no conflicts of interest regarding the publication of this paper.

\section{References}

[1] Arendorf, T.M. and Walker, D.M. (1979) Oral Candidal Populations in Health and Disease. British Dental Journal, 147, 267-272. https://doi.org/10.1038/sj.bdj.4804344

[2] Pires, F.R., Santos, E.B., Bonan, P.R., De Almeida, O.P. and Lopes, M.A. (2002) Denture Stomatitis and Salivary Candida in Brazilian Edentulous Patients. Journal of Oral Rehabilitation, 29, 1115-1119. https://doi.org/10.1046/j.1365-2842.2002.00947.x

[3] Berdicevsky, I., Ben-Aryeh, H., Szargel, R. and Gutman, D. (1980) Oral Candida of Asymptomatic Denture Wearers. International Journal of Oral Surgery, 92, 113-115. https://doi.org/10.1016/S0300-9785(80)80047-0

[4] Arendorf, T. and Addy, M. (1985) Candidal Carriage and Plaque Distribution before, during and after Removable Orthodontic Appliance Therapy. Journal of Clinical Periodontology, 12, 360-368. https://doi.org/10.1111/j.1600-051X.1985.tb00926.x

[5] Janus, M.M., Crielaard, W., Volgenant, C.M., van der Veen, M.H., Brandt, B.W. and Krom, B.P. (2017) Candida Albicans Alters the Bacterial Microbiome of Early in vitro Oral Biofilms. Journal of Oral Microbiology, 8, Article ID: 1270613. https://doi.org/10.1080/20002297.2016.1270613

[6] Jorge, A.O.C., Totti, M.A.G., de Almeida, O.P. and Scully, C. (1993) Effect of Sialoadenectomy on the Carriage of Candida Albicans in the Mouths of Rats. Journal of Oral Pathology \& Medicine, 22, 138-140. https://doi.org/10.1111/j.1600-0714.1993.tb01045.x

[7] González, G.M., Treviño-Rangel Rde, J., Palma-Nicolás, J.P., Martínez, C., González, J.G., Ayala, J., Caballero, A., Morfín-Otero, R., Rodríguez-Noriega, E., Velarde, F., Ascencio, E.P., Tinoco, J.C., Vázquez, J.A., Cano, M.A., León-Sicairos, N., González, R., Rincón, J., Elías, M.A. and Bonifaz, A. (2013) Species Distribution and Antifungal Susceptibility of Bloodstream Fungal Isolates in Paediatric Patients in Mexico: A Nationwide Surveillance Study. Journal of Antimicrobial Chemotherapy, 68, 2847-2851. https://doi.org/10.1093/jac/dkt283

[8] Miceli, M.H., Díaz, J.A. and Lee, S.A. (2011) Emerging Opportunistic Yeast Infections. The Lancet Infectious Diseases, 11, 142-151. https://doi.org/10.1016/S1473-3099(10)70218-8

[9] Pfaller, M.A., Diekema, D.J., Gibbs, D.L., Newell, V.A., Ellis, D., Tullio, V., Rodloff, A., Fu, W. and Ling, T.A. (2010) Results from the Artemis Disk Global Antifungal Surveillance Study, 1997 to 2007: A 10.5-Year Analysis of Susceptibilities of Candida Species to Fluconazole and Voriconazole as Determined by CLSI Standardized Disk Diffusion. Journal of Clinical Microbiology, 48, 1366-1377. 
https://doi.org/10.1128/JCM.02117-09

[10] Silva, S., Negri, M., Henriques, M., Oliveira, R., Williams, D.W. and Azeredo, J. (2012) Candida Glabrata, Candida Parapsilosis and Candida Tropicalis: Biology, Epidemiology, Pathogenicity and Antifungal Resistance. FEMS Microbiology Reviews, 36, 288-305. https://doi.org/10.1111/j.1574-6976.2011.00278.x

[11] Kothavade, R.J., Kura, M.M., Valand, A.G. and Panthaki, M.H. (2010) Mycological Analysis of the Oral Cavity of Patients Using Acrylic Removable Dentures. Journal of Medical Microbiology, 59, 873-880. https://doi.org/10.1099/jmm.0.013227-0

[12] Beighton, D., Ludford, R., Clark, D.T., Brailsford, S.R., Pankhurst, C.L., Tinsley, G.F., Fiske, J., Lewis, D., Daly, B. and Khalifa, N. (1995) Use of CHROmagar Candida Medium for Isolation of Yeasts from Dental Samples. Journal of Clinical Microbiology, 33, 3025-3027.

[13] Pfaller, M.A., Houston, A. and Coffmann, S. (1996) Application of CHROMagar Candida for Rapid Screening of Clinical Specimens for Candida Albicans, Candida Tropicalis, Candida Krusei, and Candida (Torulopsis) Glabrata. Journal of Clinical Microbiology, 34, 58-61.

[14] Schoofs, A., Odds, F.C., Colebunders, R., Ieven, M. and Goossens, H. (1997) Use of Specialised Isolation Media for Recognition and Identification of Candida Dubliniensis Isolates from HIV-Infected Patients. European Journal of Clinical Microbiology and Infectious Diseases, 16, 296-300. https://doi.org/10.1007/BF01695634

[15] Tamura, M., Watanabe, K., Mikami, Y., Yazawa, K. and Nishimura, K. (2001) Molecular Characterization of New Clinical Isolates of Candida Albicans and C. dubliniensis in Japan: Analysis Reveals a New Genotype of $C$. albicans with Group I Intron. Journal of Clinical Microbiology, 39, 4309-4315.

https://doi.org/10.1128/JCM.39.12.4309-4315.2001

[16] Haas, M., Grenouillet, F., Loubersac, S., Ariza, B., Pepin-Puget, L., Alvarez-Moreno, C.A., Valderrama-Beltrán, S.L., Lavergne, R.A., Le Pape, P. and Morio, F. (2016) Identification of Cryptic Candida Species by MALDI-TOF Mass Spectrometry, Not All MALDI-TOF Systems Are the Same: Focus on the C. parapsilosis Species Complex. Diagnostic Microbiology and Infectious Disease, 86, 385-386.

https://doi.org/10.1016/j.diagmicrobio.2016.08.028

[17] Lacroix, C., Gicquel, A., Sendid, B., Meyer, J., Accoceberry, I., François, N., Morio, F., Desoubeaux, G., Chandenier, J., Kauffmann-Lacroix, C., Hennequin, C., Guitard, J., Nassif, X. and Bougnoux, M.E. (2014) Evaluation of Two Matrix-Assisted Laser Desorption Ionization-Time of Flight Mass Spectrometry (MALDI-TOF MS) Systems for the Identification of Candida Species. Clinical Microbiology and Infection, 20, 153-158. https://doi.org/10.1111/1469-0691.12210

[18] Rosenvinge, F.S., Dzajic, E., Knudsen, E., Malig, S. andersen, L.B., Løvig, A., Arendrup, M.C., Jensen, T.G., Gahrn-Hansen, B. and Kemp, M. (2013) Performance of Matrix-Assisted Laser Desorption-Time of Flight Mass Spectrometry for Identification of Clinical Yeast Isolates. Mycoses, 56, 229-235. https://doi.org/10.1111/myc. 12000

[19] Landis, J.R. and Koch, G.G. (1977) The Measurement of Observer Agreement for Categorical Data. Biometrics, 33, 159-174. https://doi.org/10.2307/2529310

[20] Hatakka, K., Ahola, A.J., Yli-Knuuttila, H., Richardson, M., Poussa, T., Meurman, J.H. and Korpela, R. (2007) Probiotics Reduce the Prevalence of Oral Candida in the Elderly a Randomized Controlled Trial. Journal of Dental Research, 86, 125-130. https://doi.org/10.1177/154405910708600204

[21] Gleiznys, A., Zdanavičienè, E. and Žilinskas, J. (2015) Candida Albicans Importance 
to Denture Wearers. A Literature Review. Stomatologija, Baltic Dental and Maxillofacial Journal, 17, 54-66.

[22] Lockhart, S.R., Joly, S., Vargas, K., Swails-Wenger, J., Enger, L. and Soll, D.R. (1999) Natural Defenses against Candida Colonization Breakdown in the Oral Cavities of the Elderly. Journal of Dental Research, 78, 857-868.

https://doi.org/10.1177/00220345990780040601

[23] Hamza, O.J., Matee, M.I., Moshi, M.J., Simon, E.N., Mugusi, F., Mikx, F.H., Helderman, W.H., Rijs, A.J., van der Ven, A.J. and Verweij, P.E. (2008) Species Distribution and in Vitro Antifungal Susceptibility of Oral Yeast Isolates from Tanzanian HIV-Infected Patients with Primary and Recurrent Oropharyngeal Candidiasis. BMC Microbiology, 12, 135. https://doi.org/10.1186/1471-2180-8-135

[24] Gugnani, H.C., Becker, K., Fegeler, W., Basu, S., Chattopadhya, D., Baveja, U., Satyanarayana, S., Kalghatgi, T. and Murlidhar, A. (2003) Oropharyngeal Carriage of Candida Species in HIV-Infected Patients in India. Oropharyngeale Candida-Besiedlung Bei HIV-Infizierten in Indien. Mycoses, 46, 281-288.

https://doi.org/10.1046/j.1439-0507.2003.00896.x

[25] Mane, A., Panchvalli, S., Bembalkar, S. and Risbud, A. (2010) Species Distribution \& Antifungal Susceptibility of Oral Candida Colonising or Infecting HIV Infected Individuals. Indian Journal of Medical Research, 131, 836-838.

[26] Loster, B.W., Loster, J., Wieczorek, A. and Ryniewicz, W. (2012) Mycological Analysis of the Oral Cavity of Patients Using Acrylic Removable Dentures. Gastroenterology Research and Practice, 2012, Article ID: 951572. https://doi.org/10.1155/2012/951572

[27] Odds, F.C. and Bernaerts, R. (1994) CHROMagar Candida, a New Differential Isolation Medium for Presumptive Identification of Clinically Important Candida Species. Journal of Clinical Microbiology, 32, 923-929.

[28] Marklein, G., Josten, M., Klanke, U., Müller, E., Horré, R., Maier, T., Wenzel, T., Kostrzewa, M., Bierbaum, G., Hoerauf, A. and Sahl, H.G. (2009) Matrix-Assisted Laser Desorption Ionization-Time of Flight Mass Spectrometry for Fast and Reliable Identification of Clinical Yeast Isolates. Journal of Clinical Microbiology, 47, 2912-2917. https://doi.org/10.1128/JCM.00389-09

[29] Dhiman, N., Hall, L., Wohlfiel, S.L., Buckwalter, S.P. and Wengenack, N.L. (2011) Performance and Cost Analysis of Matrix-Assisted Laser Desorption IonizationTime of Flight Mass Spectrometry for Routine Identification of Yeast. Journal of Clinical Microbiology, 49, 1614-1616. https://doi.org/10.1128/JCM.02381-10

[30] Loster, J.E., Wieczorek, A. and Loster, B.W. (2016) Correlation between Age and Gender in Candida Species Infections of Complete Denture Wearers: A Retrospective Analysis. Clinical Interventions in Aging, 11, 1707-1714. https://doi.org/10.2147/CIA.S116658

[31] Sánchez-Vargas, L.O., Ortiz-López, N.G., Villar, M., Moragues, M.D., Aguirre, J.M., Cashat-Cruz, M., Lopez-Ribot, J.L., Gaitán-Cepeda, L.A. and Quindós, G. (2005) Oral Candida Isolates Colonizing or Infecting Human Immunodeficiency Virus-Infected and Healthy Persons in Mexico. Journal of Clinical Microbiology, 43, 4159-4162. https://doi.org/10.1128/JCM.43.8.4159-4162.2005

[32] Calderone, R.A. (2002) Introduction and Historical Perspectives. Candida and Candidiasis. In: Calderone, R., Ed., Candida and Candidiasis, ASM Press, Washington DC, $15-25$.

[33] González, G.M., Elizondo, M. and Ayala, J. (2008) Trends in Species Distribution and Susceptibility of Bloodstream Isolates of Candida Collected in Monterrey, Mexico, 
to Seven Antifungal Agents: Results of a 3-Year (2004 to 2007) Surveillance Study. Journal of Clinical Microbiology, 46, 2902-2905.

https://doi.org/10.1128/JCM.00937-08

[34] Xiao, M., Fan, X., Chen, SC., Wang, H., Sun, Z.Y., Liao, K., Chen, S.L., Yan, Y., Kang, M., Hu, Z.D., Chu, Y.Z., Hu, T.S., Ni, Y.X., Zou, G.L., Kong, F. and Xu, Y.C. (2015) Antifungal Susceptibilities of Candida Glabrata Species Complex, Candida Krusei, Candida Parapsilosis Species Complex and Candida Tropicalis Causing Invasive Candidiasis in China: 3 Year National Surveillance. Journal of Antimicrobial Chemotherapy, 70, 802-810. https://doi.org/10.1093/jac/dku460

[35] Krcmery, V. and Barnes, A.J. (2002) Non-Albicans Candida Spp. Causing Fungaemia: Pathogenicity and Antifungal Resistance. Journal of Hospital Infection, 50, 243-260. https://doi.org/10.1053/jhin.2001.1151

[36] Barchiesi, F., Orsetti, E., Gesuita, R., Skrami, E., Manso, E. and Candidemia Study Group (2016) Epidemiology, Clinical Characteristics, and Outcome of Candidemia in a Tertiary Referral Center in Italy from 2010 to 2014. Infection, 44, 205-213. https://doi.org/10.1007/s15010-015-0845-Z 